\title{
Contribution of the Portuguese Laboratory Network for the Diagnosis of Influenza A(H1N1)pdm09 Infection during the 2009/10 and 2010/11 influenza seasons
}

Portuguese Laboratory Network for the Diagnosis of Influenza Infection (raquel.guiomar@insa.min-saude.pt)

1. The members of the network are listed at the end of the article

Citation style for this article:

Portuguese Laboratory Network for the Diagnosis of Influenza Infection. Contribution of the Portuguese Laboratory Network for the Diagnosis of Influenza A(H1N1) pdmog Infection during the 2009/10 and 2010/11 influenza seasons . Euro Surveill. 2012;17(27):pii=20211. Available online: http://www.eurosurveillance.org/

ViewArticle.aspx?Articleld $=20211$

This article describes the data obtained by the Portuguese Laboratory Network, reactivated following the World Health Organization declaration of the 2009 influenza pandemic, on the diagnoses of influenza $A\left(\mathrm{H}_{1} \mathrm{~N}_{1}\right)$ pdmog infection during the pandemic (2009/10) and post-pandemic (2010/11) influenza seasons. The laboratories analysed and reported cases of influenza-like illness (ILI) and severe acute respiratory infection (SARI) to the National Influenza Reference Laboratory, which performed more detailed antigenic and genetic characterisation of the virus isolates. In 2009/10, a total of 62,089 ILI cases, distributed in two peaks, were analysed, 25,985 of which were positive for influenza $A\left(\mathrm{H}_{1} \mathrm{~N}_{1}\right)$ pdmo9. Children aged 5-14 years were the most affected. Viruses were both antigenically and genetically similar to the pandemic strain A/California/7/2009, included in the 2009/10 pandemic vaccine. During the post-pandemic season, 1,496 ILI cases were tested for influenza $A\left(\mathrm{H}_{1} \mathrm{~N}_{1}\right)$ pdmo9, 572 of which were positive. Infection was mainly diagnosed in adolescent and adults. Although the 2010/11 viruses remained antigenically similar to $\mathrm{A} /$ California/7/2009, increased genetic variation was observed. During the two seasons, two viruses with the neuraminidase $\mathrm{H} 275 \mathrm{Y}$ amino acid substitution, associated with oseltamivir resistance, were detected. The Laboratory Network made an important contribution to the description of the influenza activity in the two seasons.

\section{Introduction}

Although epidemics of influenza occur almost every year in temperate climates, the rates and severity of illness can vary substantially between seasons [1]. Every winter season influenza infection is associated with a considerable number of fatal cases especially in elderly people with chronic diseases [2]. Recent studies from different countries, including Portugal $[3,4]$, estimate that $90 \%$ of excess deaths due to seasonal influenza occur in the elderly ( $\geq 65$ years) who are at higher risk due to comorbidities. In Portugal, the seasons with higher excess mortality were associated with the predominant circulation of influenza $\mathrm{A}\left(\mathrm{H}_{3}\right)$ viruses, while predominant circulation of type $B$ viruses had a lower impact in mortality [4].

In March 2009 a new influenza virus circulating in humans emerged in Mexico. The new influenza $A\left(\mathrm{H}_{1} \mathrm{~N}_{1}\right)$ pdmo9, as it was later called, started disseminating throughout the world, giving rise to the first influenza pandemic of the 21st century, and appears to have been introduced in Europe by travellers returning from Mexico and by their contacts. Following the detection of the initial imported cases, there was a spring and summer wave of transmission which affected several European countries. Transmission accelerated again after the re-opening of schools, this time affecting all countries, and lead to an early autumn/winter wave [5].

Since 1990 and until the 2009/10 winter season, influenza surveillance in Portugal had been carried out in the context of a National Influenza Surveillance Programme, coordinated by the National Influenza Reference Laboratory in cooperation with the Department of Epidemiology of the National Institute of Health and the General-Directorate of Health [6]. Activities within the Programme included collecting, analysing and reporting clinical, epidemiological and virological data on influenza-like illness (ILI), integrated from a sentinel network of general practitioners and a non-sentinel network of emergency units located at hospitals and health centres from the National Health System. As it was well established, the Programme proved essential for monitoring the pandemic in Portugal [7], particularly in the pre-pandemic phases when all suspected cases were referred to reference hospitals and the laboratory analysis was carried out centrally at the National Influenza Reference Laboratory, as defined in the National Contingency Plan for an Influenza Pandemic [8]. 
After the beginning of the pandemic, and to cope with the increasing number of diagnoses requested by the National Health System, the Portuguese Laboratory Network for the Diagnosis of Influenza was activated [9]. This Network had originally been established in 2006 with eight laboratories involved [10], after the emergence of human cases of influenza $\mathrm{A}\left(\mathrm{H}_{5}\right)$ virus infection, with the purpose of supporting, at the national level, the laboratory diagnosis of infection by a new influenza pandemic virus, but was subsequentially de-activated in the absence of zoonotic transmission of new emergent influenza viruses. In May 2009, professionals from these laboratories began training in molecular methodologies for detection of the new influenza $A\left(\mathrm{H}_{1} \mathrm{~N}_{1}\right)$ pdmog virus. In the following two months, additional laboratories were involved and by the end of July 2009, all 13 members of the Network were able to perform those methods. Since then, the information generated, particularly that concerning the detection and molecular characterisation of influenza viruses associated with severe disease, has been integrated into the National Influenza Surveillance Programme and improved the understanding of the influenza activity in Portugal.

The National Influenza Reference Laboratory, which coordinates the Portuguese Laboratory Network for the Diagnosis of Influenza, also analysed and confirmed suspected cases of antiviral resistance. The majority of globally circulating influenza $A\left(\mathrm{H}_{1} \mathrm{~N}_{1}\right)$ pdmog viruses during the two seasons were still sensitive to oseltamivir and zanamivir; oseltamivir-resistant influenza $A\left(\mathrm{H}_{1} \mathrm{~N}_{1}\right)$ pdmog strains were found only sporadically and were associated with the substitution $\mathrm{H}_{275} \mathrm{Y}$ in neuraminidase gene [11].

This study describes the influenza activity during the 2009/10 and 2010/11 influenza seasons, based on the information collected through the Portuguese Laboratory Network for the Diagnosis of Influenza.

\section{Methods}

\section{Surveillance period}

The influenza period 2009/10 was epidemiologically different from a normal influenza winter season. For this reason, it was decided to consider as the 2009/10 pandemic season the period from week 17, 2009 to week 20, 2010. This particular period was chosen because the first influenza-positive case attributed to influenza $\mathrm{A}\left(\mathrm{H}_{1} \mathrm{~N}_{1}\right)$ pdmog infection was observed in week 17, 2009. During the 2010/11 season the laboratory Network was active during the usual surveillance period between October and May (weeks 40, 2010 through 20, 2011).

All 13 laboratories reported cases during the pandemic $2009 / 10$ season. During the $2010 / 11$ winter, only nine participated in the reporting of cases with respiratory disease.

\section{FIGURE 1}

Laboratories of the Portuguese Laboratory Network for the Diagnosis of Influenza Infection

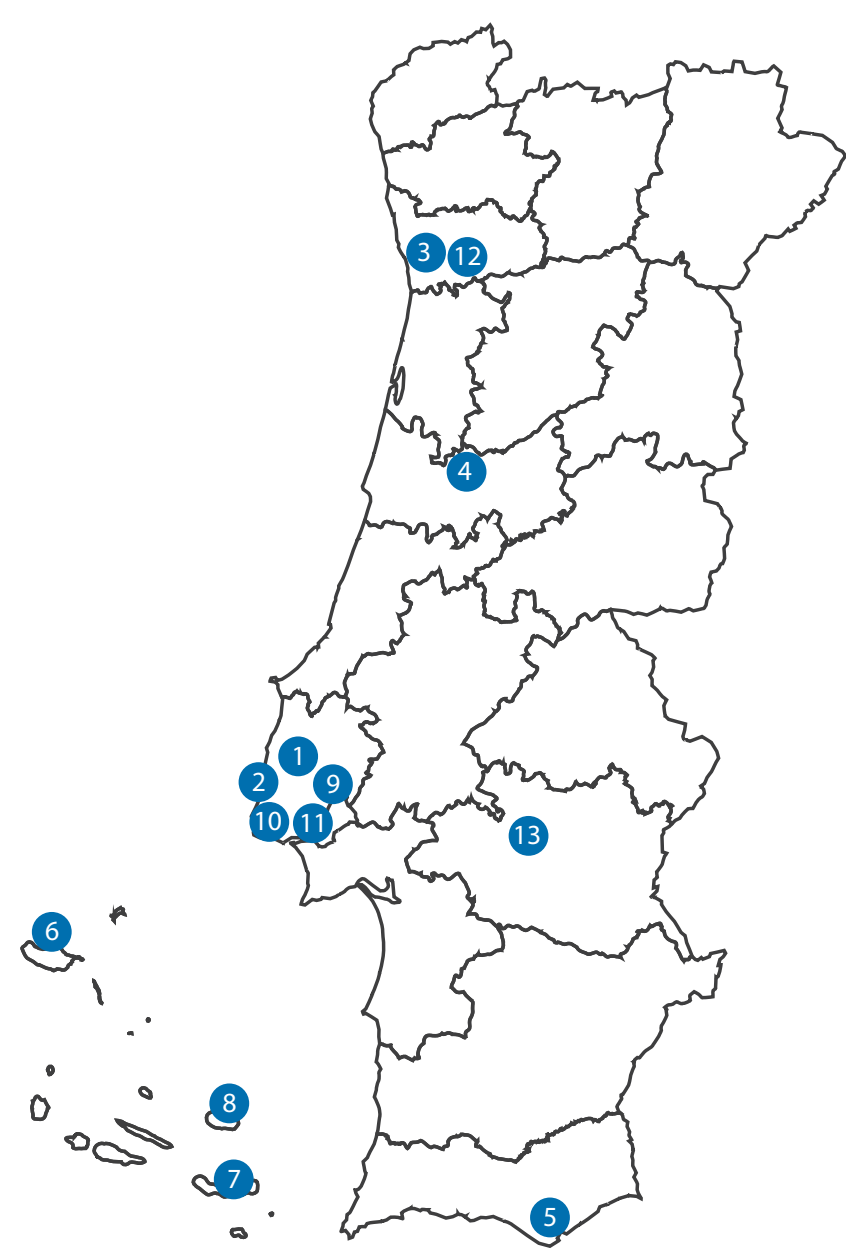

(1) Instituto Nacional de Saúde Doutor Ricardo Jorge, I.P.; (2) Hospital de Curry Cabral; (3) Centro Hospitalar de S. João, E.P.E.; (4) Hospitais da Universidade de Coimbra, E.P.E.; (5) ARS do Algarve, I.P. - Laboratório Regional de Saúde Pública Laura Ayres; (6) Hospital Central do Funchal, E.P.E.; (7) Hospital do Divino Espírito Santo de Ponta Delgada, E.P.E.; (8) Hospital de Santo Espírito de Angra do Heroísmo, E.P.E.; (9) Instituto de Medicina Molecular da Faculdade de Medicina da Universidade de Lisboa; (10) Centro Hospitalar Lisboa Norte, E.P.E.; (11) Centro Hospitalar Lisboa Central, E.P.E.; (12) Centro Hospitalar do Porto, E.P.E.; (13) Hospital do Espírito Santo de Évora, E.P.E.

The Portuguese Laboratory Network for the Diagnosis of Influenza Infection

The Portuguese Laboratory Network for the Diagnosis of Influenza Infection is currently composed of 13 laboratories from reference hospitals on mainland Portugal and the islands (Figure 1).

The main objective of this Network during the 2009/10 and $2010 / 11$ influenza seasons was to carry out the laboratory diagnosis of influenza $A\left(\mathrm{H}_{1} \mathrm{~N}_{1}\right)$ pdmo9 infection requested by the National Health Service. Currently, diagnostic capabilities include detection not only of influenza $A\left(\mathrm{H}_{1} \mathrm{~N}_{1}\right)$ pdmog virus but also influenza $B$ virus (six laboratories), influenza $A\left(\mathrm{H}_{1}\right)$ and 
$A\left(H_{3}\right)$ seasonal viruses (four laboratories), and other infectious agents associated with respiratory disease: adenovirus, coronavirus, rhinovirus, respiratory syncytial virus, metapneumovirus, parainfluenza virus, Haemophilus influenzae and Streptococcus pneumoniae (three laboratories).

\section{Cases and variables studied}

During the two seasons, ILI cases from hospital emergency units as well as from other primary healthcare units, and hospitalised cases of severe acute respiratory infection (SARI), were reported through a common Internet-based database or by an Excel spreadsheet sent to the National Influenza Reference Laboratory. Published case definitions were applied [12]. The notification form included clinical and epidemiological information, such as demographic data, signs and symptoms, underlying conditions and information on vaccination and antiviral therapy.

\section{Laboratory analysis}

For laboratory diagnosis and further virological characterisation, nasopharyngeal swabs, or combined nasopharyngeal/oropharyngeal swabs, were collected into a suitable transport medium. Different real-time RT-PCR methodologies and platforms were used within the Network for the laboratory diagnosis of influenza infection.

Upon request from the Network, the National Influenza Reference Laboratory performed additional diagnoses (typing, subtyping and lineage determination by realtime PCR of other influenza viruses), viral isolation [13] and antigenic and genetic characterisation of viral isolates, according to the procedures adopted from the manual for the laboratory diagnosis and virological surveillance of influenza published by the World Health Organisation (WHO) [1] and adapted from the United States Centers for Disease Prevention and Control (CDC) [14].

Influenza virus isolates were characterised antigenically by haemagglutination inhibition tests (HAI) using antisera and reference virus strains distributed by the WHO Collaborating Centre in Atlanta. Selected isolates were sent to the WHO Collaborating Centre in London for further study. Genetic analysis of isolates was carried out by sequencing the haemagglutinin (HA) gene encoding the $\mathrm{HA}_{1}$ subunit, using primer sequences available from CDC [14], for a subset of approximately $25 \%$ of isolates encompassing the beginning, middle and end of each season. Sequences are available from the GenBank database. Phylogenetic analysis was performed with MEGA Software [15]. HA1 sequences from reference strains used in the genetic analysis were obtained from the EpiFlu database of the Global Initiative on Sharing Avian Influenza Data (GISAID) (Table 1).

The detection of antiviral resistance was performed by phenotypical assays, such as the neuraminidase inhibition assay, and by neuraminidase gene sequencing $[1,14]$.

\section{Statistical analysis}

The probability of occurrence of an influenza case in the presence of the signs/symptoms considered for definition of ILI was estimated by calculating the individual crude odds ratios, and the respective $95 \%$ confidence intervals, of reporting each sign/symptom in both influenza cases (laboratory-confirmed) and ILI cases (influenza-negative result).

\section{Results}

\section{9/10 season}

In Portugal, a total of $62,089 \mathrm{ILI}$ cases were reported through the Laboratory Network during the 2009/10 pandemic season. Two peaks were observed, one occurring towards the end of the summer 2009 and another during November and December of the same year (Figure 2).

The first case of influenza $A\left(\mathrm{H}_{1} \mathrm{~N}_{1}\right)$ pdmog infection in Portugal was detected during week 17, 2009, when seasonal influenza viruses, linked to the previous winter season, were still circulating. A total of 25,985 laboratory-confirmed influenza cases were found, over $99 \%$ of which were associated with the new pandemic strain $A\left(\mathrm{H}_{1} \mathrm{~N}_{1}\right)$ pdmog. Other influenza viruses were also found: 190 non-subtyped influenza $A$ viruses, 15 seasonal influenza $A\left(\mathrm{H}_{1}\right)$ viruses, 10 influenza $A\left(\mathrm{H}_{3}\right)$ and 31 influenza $B$ viruses. A first peak in influenza cases was observed during week 33 with 1,145 influenza $\mathrm{A}\left(\mathrm{H}_{1} \mathrm{~N}_{1}\right)$ pdmog cases $(45.9 \%$ of all ILI cases notified in week 33). Most of the cases during the first wave were detected in the south of Portugal and in the main urban areas (Lisbon and Oporto). The first wave was followed by a second wave with cases all over the country (mainland and islands) and with a peak during week 46 with 3,266 influenza $\mathrm{A}\left(\mathrm{H}_{1} \mathrm{~N}_{1}\right)$ pdmog cases (60.2\% of all ILI cases notified in week 46$)$.

Although the majority of notifications occurred in the population with 15 to 44 years of age $(n=21,267$; $34.3 \%$ of all ILI cases), the highest proportion of influenza-positive results was observed in children aged between five and 14 years $(n=10,093 ; 38.8 \%$ of all influenza $\mathrm{A}\left(\mathrm{H}_{1} \mathrm{~N}_{1}\right)$ pdmog-positive cases) (Table 2). The lowest number of reported cases $(n=1,095 ; 1.8 \%)$ and influenza-positive results $(n=515 ; 2.0 \%)$ were reported in the elderly. The highest proportion of influenza $A\left(\mathrm{H}_{1} \mathrm{~N}_{1}\right)$ pdmo9-positive results within any one age group $(64.0 \%)$ was observed in children aged 5-14 years.

Eighty influenza strains were isolated and antigenically characterised, 43 of which were also genetically analysed (HA gene). The 80 influenza $A\left(\mathrm{H}_{1} \mathrm{~N}_{1}\right)$ pdmog strains isolated were antigenically homogeneous and showed high similarity with influenza A/ California/7/2009 (strain included in the pandemic 


\begin{tabular}{|c|c|c|c|c|c|c|c|c|c|c|c|c|c|c|c|c|c|c|c|c|c|c|c|c|c|}
\hline & & & & & & & & & & & & & & 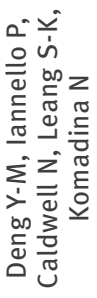 & & & & & & & & 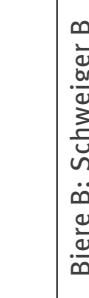 & 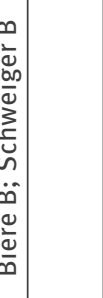 & & \\
\hline 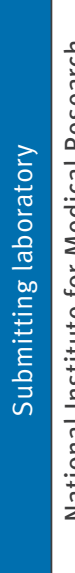 & 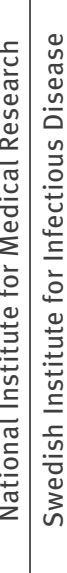 & : & 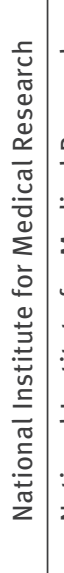 & 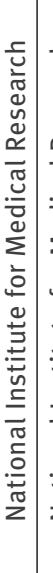 & 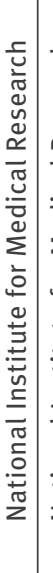 & 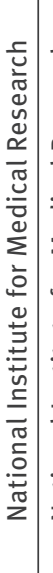 & 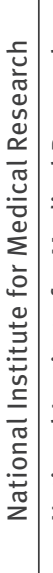 & 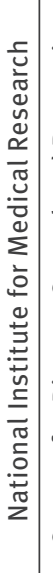 & 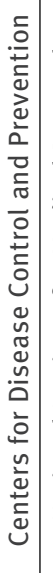 & 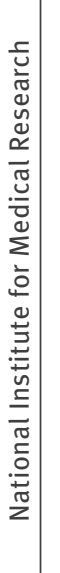 & 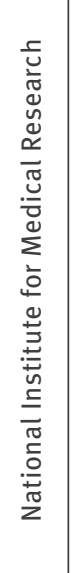 & 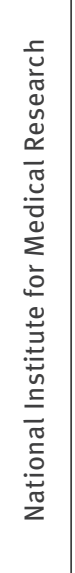 & 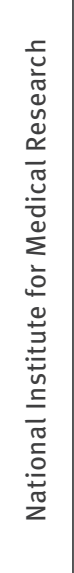 & 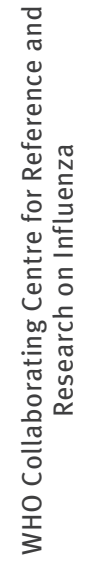 & 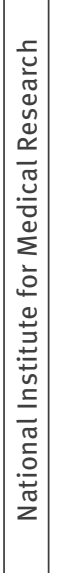 & 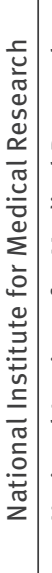 & 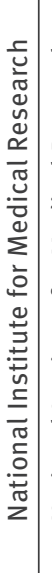 & 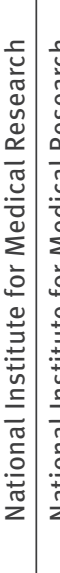 & 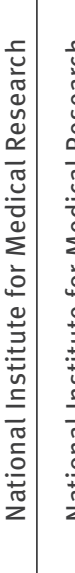 & 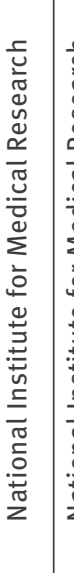 & 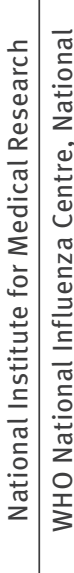 & 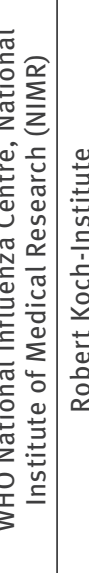 & 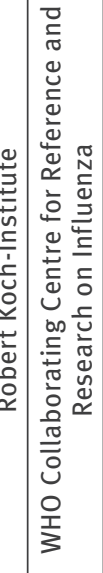 & 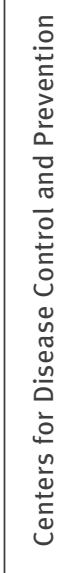 & 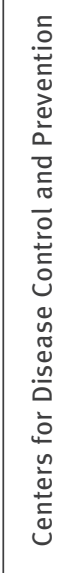 \\
\hline 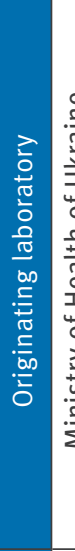 & 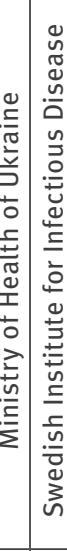 & : & 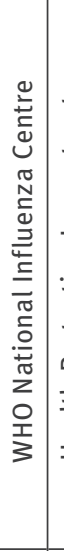 & 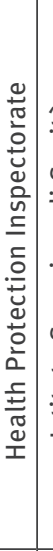 & 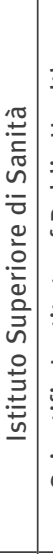 & 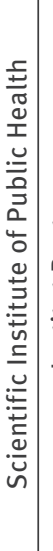 & 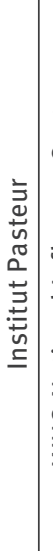 & 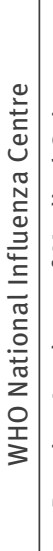 & 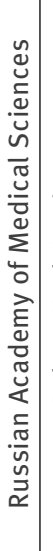 & 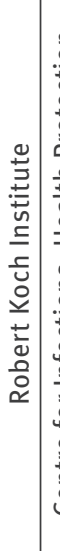 & 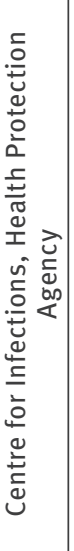 & 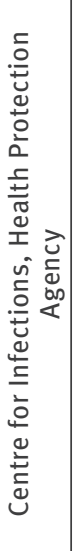 & 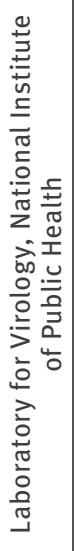 & 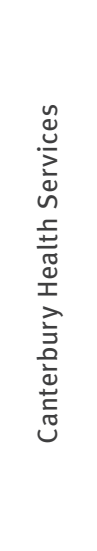 & 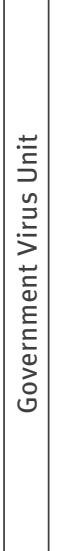 & 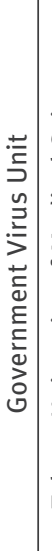 & 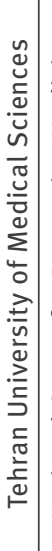 & 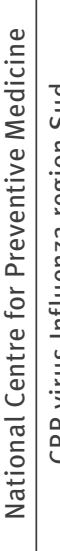 & 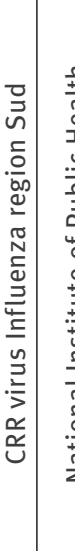 & 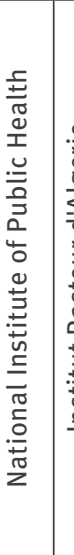 & 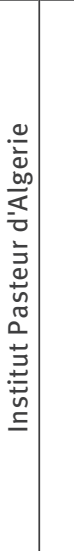 & 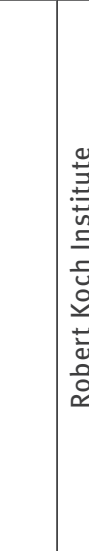 & 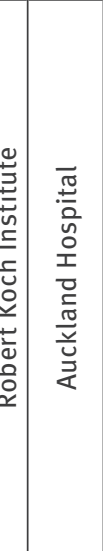 & 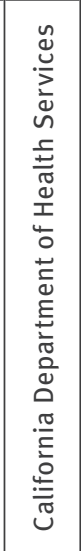 & \\
\hline 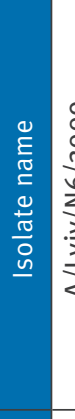 & 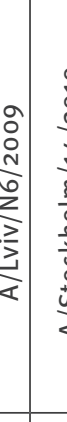 & 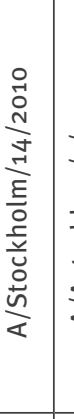 & 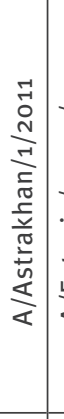 & 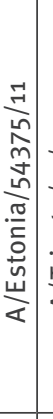 & 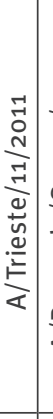 & 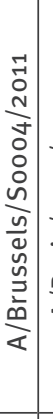 & 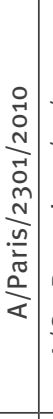 & 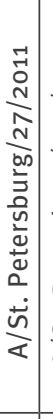 & 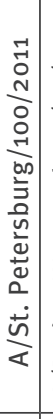 & 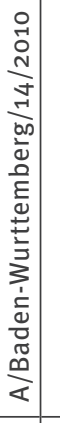 & 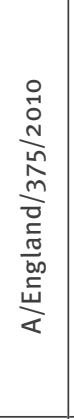 & 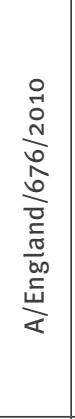 & 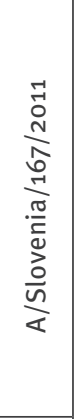 & 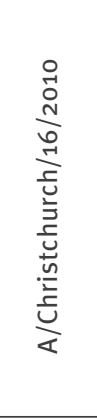 & 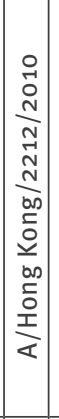 & 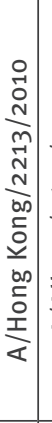 & 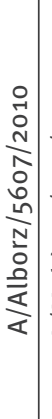 & 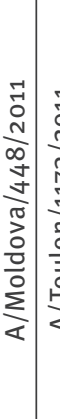 & 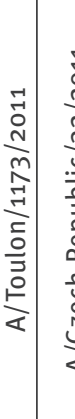 & 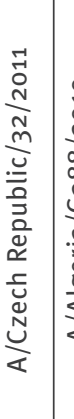 & 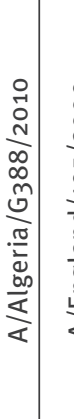 & 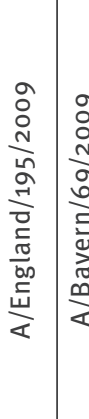 & 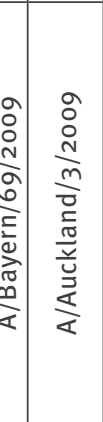 & 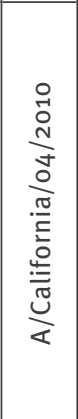 & 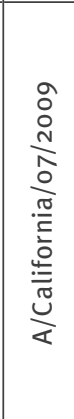 \\
\hline 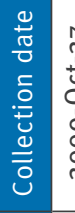 & 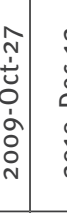 & 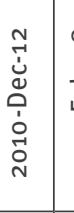 & 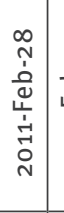 & 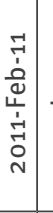 & 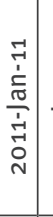 & 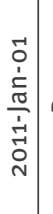 & 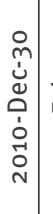 & 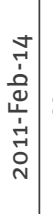 & 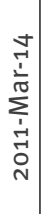 & 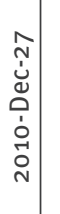 & $\begin{array}{l}\vec{m} \\
\dot{\tilde{\Xi}} \\
\stackrel{0}{0} \\
\dot{0} \\
\dot{N}\end{array}$ & $\begin{array}{l}0 \\
\stackrel{\tilde{U}}{0} \\
\stackrel{\Delta}{0} \\
\dot{0} \\
\dot{N}\end{array}$ & 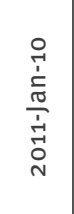 & $\begin{array}{l}\frac{N}{I} \\
\frac{N}{\grave{D}} \\
\dot{0} \\
\stackrel{N}{N}\end{array}$ & 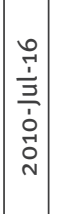 & 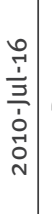 & $\begin{array}{l}0 \\
0 \\
\dot{d} \\
0 \\
0 \\
0 \\
0 \\
0 \\
\end{array}$ & 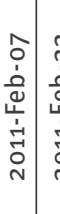 & 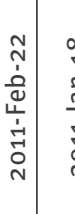 & 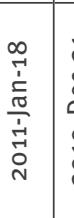 & 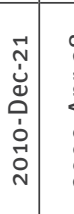 & 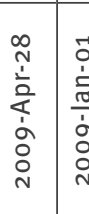 & 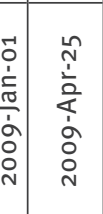 & $\begin{array}{l}\overrightarrow{7} \\
\dot{1} \\
\dot{0} \\
\dot{1} \\
\dot{0} \\
\dot{N}\end{array}$ & 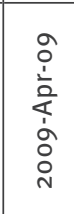 \\
\hline 辛 & 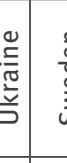 & 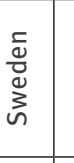 & & 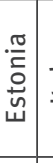 & 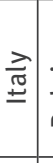 & 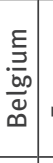 & 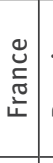 & 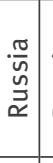 & 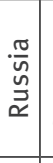 & 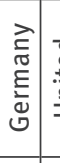 & 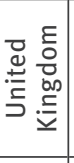 & 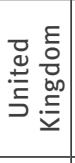 & $\begin{array}{l}\frac{.}{\bar{\sigma}} \\
\frac{\bar{d}}{0} \\
\frac{0}{n}\end{array}$ & 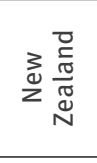 & 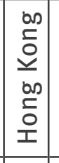 & 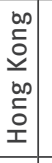 & & 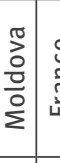 & 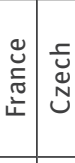 & 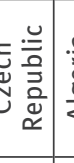 & 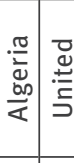 & 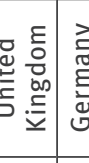 & 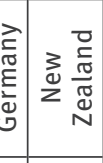 & 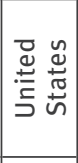 & 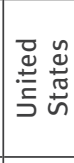 \\
\hline 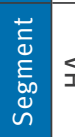 & 臣 & 至 & 吕 & $\overleftrightarrow{\mathbf{x}}$ & $\underline{\mathbf{I}}$ & 坕 & $\underline{\mathbf{I}}$ & $\Phi$ & $\Phi$ & 哇 & 吕 & $\stackrel{\mathbb{1}}{ }$ & 坕 & 至 & $\mid \underline{1}$ & I & & 至 & 至 & 吕 & エ & 吕 & 坕 & 王 & 至 \\
\hline 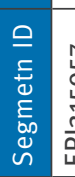 & 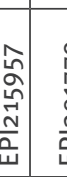 & 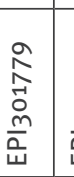 & 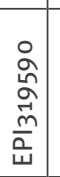 & & & & & & & $\begin{array}{l}\vdots \\
\vdots \\
\vdots \\
\dot{m} \\
\frac{m}{4}\end{array}$ & $\begin{array}{l}\text { 寽 } \\
\stackrel{0}{\circ} \\
\stackrel{m}{\frac{m}{\omega}}\end{array}$ & $\begin{array}{l}\hat{o} \\
\stackrel{\circ}{a} \\
\stackrel{m}{\omega} \\
\dot{\omega}\end{array}$ & $\begin{array}{l}\tilde{0} \\
0 \\
\stackrel{\circ}{\omega} \\
\frac{m}{\omega}\end{array}$ & $\begin{array}{l}\hat{O} \\
\text { D } \\
\infty \\
\hat{N} \\
\frac{N}{\omega}\end{array}$ & 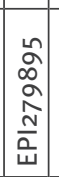 & & & 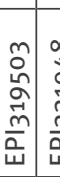 & 告 & 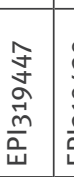 & 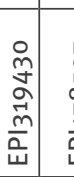 & 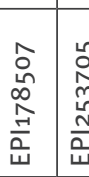 & 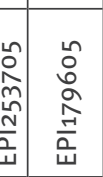 & 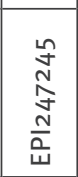 & 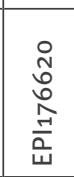 \\
\hline
\end{tabular}




\section{FIGURE 2}

Figure 2. Weekly distribution of influenza-like illness and influenza A(H1N1)pdm09-positive cases, Portugal, 2009/10 and $2010 / 2011$ seasons $(n=63,585)$
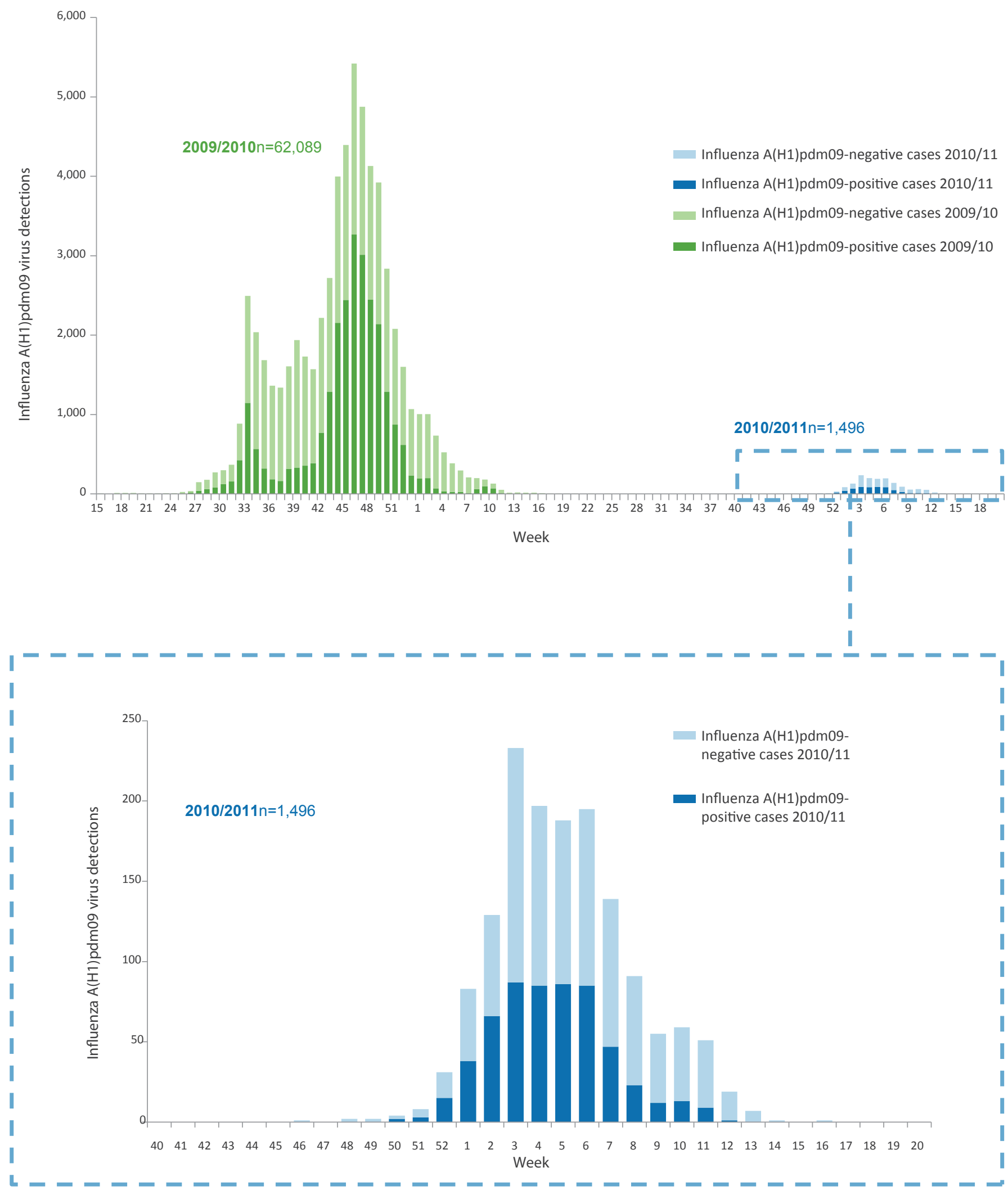
Distribution of influenza A(H1N1)pdm09 cases, by age group, Portugal, 2009/10 and 2010/11 seasons (n=63,585)

\begin{tabular}{|c|c|c|c|c|}
\hline \multirow{2}{*}{$\begin{array}{l}\text { Age group } \\
\text { (years) }\end{array}$} & \multicolumn{2}{|r|}{$2009 / 10$} & \multicolumn{2}{|r|}{$2010 / 11$} \\
\hline & Number of cases & $\begin{array}{c}\text { Influenza } \mathrm{A}\left(\mathrm{H}_{1} \mathrm{~N}_{1}\right) \text { pdmo9 } \\
\text { (\% positive within the age group) }\end{array}$ & Number of cases ${ }^{a}$ & $\begin{array}{c}\text { Influenza } \mathrm{A}\left(\mathrm{H}_{1} \mathrm{~N}_{1}\right) \text { pdmog } \\
\text { (\% positive within the age group) }\end{array}$ \\
\hline $0-4$ & 15,189 & $4,042(26.6 \%)$ & 192 & $48(25.0 \%)$ \\
\hline 5-14 & 15,765 & $10,093(64.0 \%)$ & 47 & $19(40.4 \%)$ \\
\hline $15-44$ & 21,267 & $9,216(43.3 \%)$ & 637 & $286(44.9 \%)$ \\
\hline $45-64$ & 6,132 & $1,831(29.9 \%)$ & 402 & $173(43.0 \%)$ \\
\hline$\geq 65$ & 2,641 & $288(10.9 \%)$ & 207 & $41(19.8 \%)$ \\
\hline Information unavailable & 1,095 & $515(47.0 \%)$ & 11 & $5(45.5 \%)$ \\
\hline Total & 62,089 & $25,985(41.9 \%)$ & 1,496 & $572(38.2 \%)$ \\
\hline
\end{tabular}

a Although 1,512 cases were reported in 2010/11, only 1,496 were tested for influenza $A\left(\mathrm{H}_{1} \mathrm{~N}_{1}\right) \mathrm{pdmo9}$. h.

vaccine of the season) and with the later pandemic influenza $A\left(\mathrm{H}_{1} \mathrm{~N}_{1}\right)$ viruses $A / B a y e r n / 69 / 2009$ and $A /$ Lviv/N6/2009 (data not shown).

All sequenced HA1 segments from 2009/10 isolates were similar to influenza A/California/7/2009 (Figure 3). Compared to the vaccine strain, with the exception of influenza A/Lisboa/31/2009 and A/Lisboa/35/2009, all isolates contained the P83S amino acid substitution and grouped into clade 7 (S203T substitution in the $\mathrm{Ca}$ antibody binding site of $\mathrm{HA1}$ ), described in the literature early in the pandemic [16]. Additional mutations were found in the $\mathrm{HA}_{1}$ antigenic sites: $\mathrm{G}_{170 R}$ (A/Lisboa/65/2009), R205K (A/Lisboa/73/2009), D222E (observed in 18 isolated viruses) and S74G (A/Lisboa/171/2009). The sequences are available from GenBank with the following accession numbers: CYo67801, CYo67804, CY067807, CY067810, CYo67819, CYo67822, CY067825, CY067828, CY067831, CYo67834, CYo67837, CY067840, CYo67843, CYo67846, CYo67849, CYo67852, CYo67855, CYo67858, CYo67861, CY067864, CYo67867, CYo67870, CYo67873, CYo67876, CYo67879, CYo67882, CYo67885, CYo67888, CYo67891, CYo67894, CY067897, CYo67900, CY067903, CYo67906, CYo67909, CYo67912, CYo67915, CYo67918, CY067765, CYo67789, CYo67792, CYo67795, CYo67798.

The amino acid substitution $\mathrm{H}_{275} \mathrm{Y}$ associated with oseltamivir resistance was found in the neuraminidase of one strain (A/Lisboa/171/2009), which was proved to be resistant by phenotypic assays.

\section{0/11 season}

Nine laboratories reported a total of 1,512 ILI cases, from week 40, 2010 to week 16,2011 , with a peak of 236 cases $(15.6 \%)$ observed in week 3, 2011. Influenza $\mathrm{A}\left(\mathrm{H}_{1} \mathrm{~N}_{1}\right)$ pdmog was tested for in 1,496 cases, 572 $(38.2 \%)$ of which were positive. The peak of laboratory-confirmed $\mathrm{A}\left(\mathrm{H}_{1} \mathrm{~N}_{1}\right)$ pdmog-associated cases was also registered in week 3 of $2011(n=87 ; 37.3 \%$ of all ILI cases notified in week 3 ).

The majority of notifications ( $n=637 ; 42.6 \%$ of all ILI cases) and the higher proportion of influenza $A\left(\mathrm{H}_{1} \mathrm{~N}_{1}\right)$ pdmog cases ( $n=286,50.0 \%$ of all positive results) occurred in the population between 15 and 44 years of age (Table 2). The lowest number of reported cases $(n=47 ; 3.1 \%)$ and pandemic influenza-positive results $(n=19 ; 3.3 \%)$ were reported in children aged 5 to 14 years. The higher proportion of influenza $A\left(\mathrm{H}_{1} \mathrm{~N}_{1}\right)$ pdmog-positive results within an age group was observed in older children and adults (15-44 years: $44.9 \%$; $45-64$ years: $43.0 \%$ ).

Following a single request from the Network in that season for additional characterisation at the National Influenza Reference Laboratory, the HA1 nucleotide sequence of one influenza $\mathrm{A}\left(\mathrm{H}_{1} \mathrm{~N}_{1}\right)$ pdmog detected in a clinical specimen was determined (Figure 3). This virus clustered in the genetic clade of $A /$ Astrakhan/1/2011, one of four genetic clades circulating in Portugal during the 2010/11 season (data not shown), and contained the P83S, I321V and S203T amino acid substitutions common to all 14 viruses analysed in that season, and the substitutions D97N, R205K, I216V and V249L characteristic of the $A / A s t r a k h a n / 1 / 2011$ group. This virus also presented the $\mathrm{H}_{275} \mathrm{Y}$ mutation in the neuraminidase, associated with oseltamivir resistance.

From the clinical symptoms analysed, fever was the one that revealed a higher risk of being observed in an influenza case (crude OR: 3.3; 95\% Cl: 1.3-8.0) (Table 3).

Three laboratories also performed the differential diagnosis of respiratory infection in 284 cases, $12.7 \%$ of which were positive for other infectious agents (one adenovirus, three coronaviruses, one human metapneumovirus, 12 rhinoviruses, nine respiratory syncytial 


\section{FIGURE 3}

Maximum likelihood phylogenetic tree of HA1 nucleotide sequences from influenza A(H1N1)pdm09 viruses,

Portugal, 2009/10 and 2010/2011 seasons $(n=44)$.

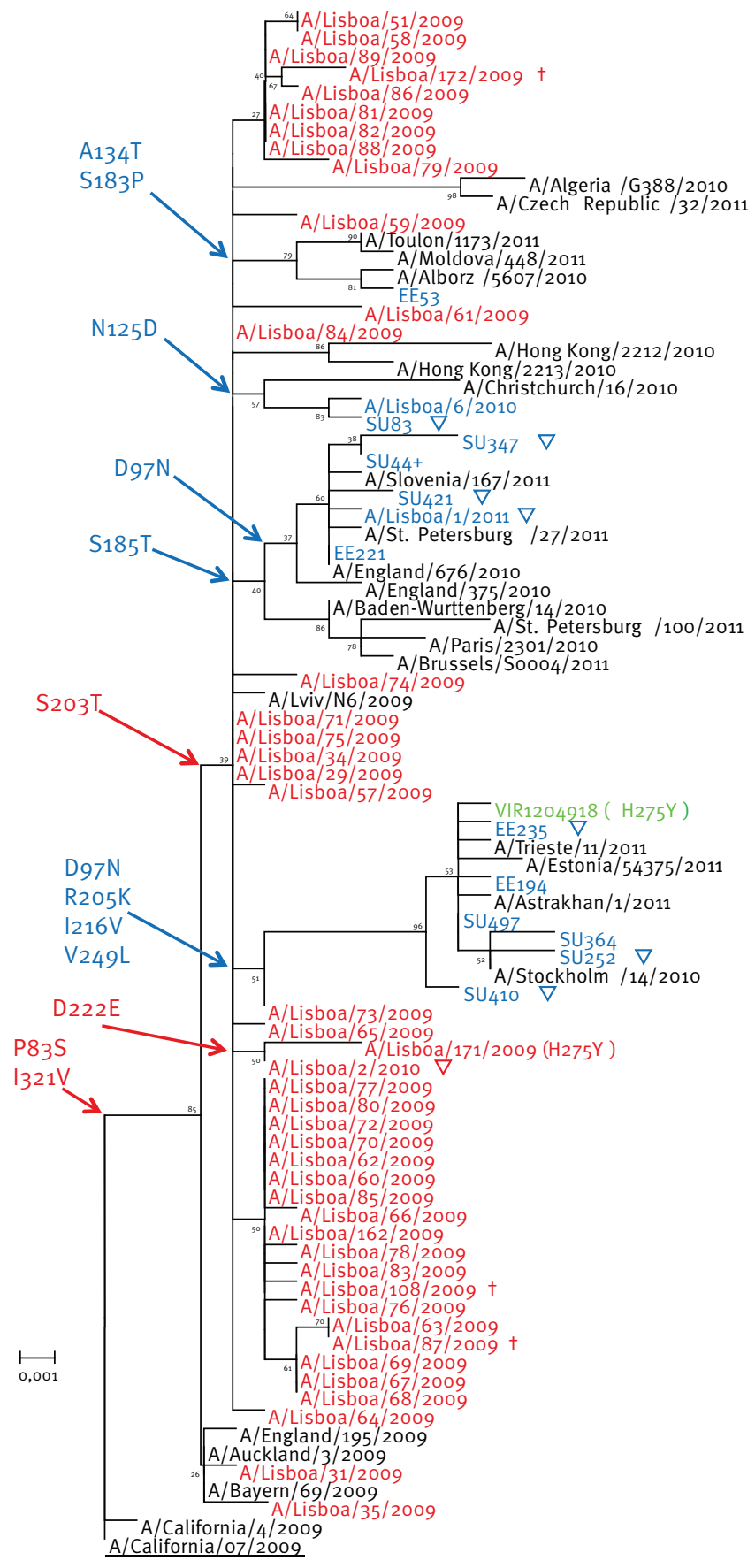

The 43 strains and amino acid substitutions from the 2009/10 pandemic season are represented in red. The virus detected in a clinical specimen from the $2010 / 11$ season is represented in green. The other 14 viruses detected in clinical specimens, which were circulating in Portugal during the $2010 / 11$ season (data from the National Influenza Surveillance Programme), and amino acid substitutions found are represented in blue. Reference strains (obtained from GISAID`s EpiFlu database) are in black and the vaccine strain is underlined; $†$ indicates cases with fatal outcome. $\nabla$ indicates cases vaccinated at least 14 days before onset of symptoms. Bootstrap values above 50 are shown (500 replicates). virus, one $H$. influenzae, one $S$. pneumoniae and seven mixed infections).

\section{Discussion}

Facing the circulation of a new influenza $A\left(\mathrm{H}_{1} \mathrm{~N}_{1}\right)$ pdmog virus and the potential threat to the population and the healthcare system, and the resulting increased awareness and diagnostic requirements, the total number of ILI cases reported and analysed in our country during the 2009/10 winter was much higher than in previous influenza seasons. Cases with laboratory-confirmed diagnosis for influenza $\mathrm{A}\left(\mathrm{H}_{1} \mathrm{~N}_{1}\right)$ pdmog increased mainly due to wide sampling criteria and to rapid diagnostic tests.

Given the considerable number of diagnoses performed in different settings, both in primary care and hospitals, the Laboratory Network has made an important contribution to improve the surveillance of influenza activity in Portugal. Regarding the pattern of disease, the data collected through the Laboratory Network support the data obtained from the National Influenza Surveillance Programme [7] and suggest that the 2009/10 pandemic was similar to the previous influenza season in intensity and geographical distribution. Seasonal viruses were replaced by the new influenza $A\left(\mathrm{H}_{1} \mathrm{~N}_{1}\right)$ pdmog strain, which caused disease particularly in young children, consistent with the data from all European countries where children under the age of 14 years were affected the most [5].

Using the data generated from the Laboratory Network it was possible to better characterise the beginning of the pandemic season, as the early cases were mainly reported to the reference hospitals, dedicated to the isolation of patients in an attempt to contain the virus and contribute to the management of the infection, as defined in the National Contingency Plan for an Influenza Pandemic [8]. During the mitigation phase of the pandemic, when the new pandemic strain had disseminated throughout the world, both the Laboratory Network and the National Influenza Surveillance Programme contributed to the clinical, epidemiological and virological characterisation of the infection.

The pandemic season was characterised by two waves, one in the summer and another during the winter. A very similar pattern was observed in Spain and other European countries [5,17]. In Portugal the pandemic influenza $A\left(\mathrm{H}_{1} \mathrm{~N}_{1}\right)$ pdmog virus was first detected on 4 May, soon after the first case confirmed in Spain (27 April 2009) and in Europe (13 April 2009). Data from Spain and combined data for Europe show the first peak of influenza cases in 2009 during weeks 31 and 30, and the second peak during weeks 47 and 46, respectively $[5,17]$. The 2009 pandemic was considered a mild one for Europe, with little impact on services outside the health sector [5].

In the 2010/11 season, the first post-pandemic season, the number of laboratory diagnosis requests 
Correlation between presence of symptoms/signs considered for clinical definition of influenza-like illness and influenza cases, Portugal, 2009/10 and 2010/11 seasons $(\mathrm{n}=63,585)$

\begin{tabular}{|c|c|c|c|c|c|c|}
\hline \multirow{2}{*}{ Sign/symptom } & \multicolumn{3}{|c|}{$2009 / 10$} & \multicolumn{3}{|c|}{$2010 / 11$} \\
\hline & Positive cases (\%) & OR (crude) & $\mathrm{Cl}(95 \%)$ & Positive cases (\%) & OR (crude) & $\mathrm{Cl}(95 \%)$ \\
\hline Fever $\left(\geq 38^{\circ} \mathrm{C}\right)$ & 44.1 & 1.3 & $1.3-1.4$ & 43.3 & $3 \cdot 3$ & $1.3-8.0$ \\
\hline Cough & 47.4 & 2.4 & $2.3-2.5$ & 39.6 & 1.0 & $0.4-2.5$ \\
\hline Sore throat & 42.1 & 1.0 & $0.4-2.4$ & $44 \cdot 7$ & 1.4 & $0.7-3.0$ \\
\hline Respiratory difficulty & 29.4 & 0.5 & $0.4-0.5$ & 50.0 & 1.0 & $0.3-2.8$ \\
\hline Contact with another influenza patient & 63.6 & 3.6 & $3.1-4.1$ & NA & NA & NA \\
\hline Myalgia & 47.2 & 1.4 & $1.4-1.5$ & NA & NA & NA \\
\hline Headache & 50.4 & 1.8 & $1.8-1.9$ & NA & NA & NA \\
\hline Sudden onset & NA & NA & NA & 40.0 & 1.1 & $0.6-2.3$ \\
\hline
\end{tabular}

$\mathrm{Cl}$ : confidence interval; NA: not applicable; OR: odds ratio.

The symptoms/signs that revealed an elevated risk of being observed in an influenza case are represented in bold. Number of ILI cases reporting information differed for each sign/symptom; only valid records (reporting presence or absence of signs/symptoms and with a valid laboratory result) were considered in the calculation of individual crude odds ratios. The presence of a sign/symptom is compared between an ILI case and an influenza case.

dramatically decreased due to tighter criteria for sampling, although the actual ILI incidence rates were in the same order of magnitude as in the two previous seasons. The highest incidence rates for the seasons 2008/09, 2009/10 and 2010/11 were 199.5, 133.7 and 121.1 cases per 100,000 inhabitants, respectively [7]. Hence, the 41-fold difference in reporting cases between the pandemic and post-pandemic season, as seen in the data from the Laboratory Network, is due to the dramatic reduction in requests for diagnostics. The Network being a non-sentinel component of the National Influenza Surveillance Programme and inactive before the start of the 2009 pandemic, 2008/09 data for comparison are not available.

According to the National Influenza Surveillance Programme, the influenza $A\left(\mathrm{H}_{1} \mathrm{~N}_{1}\right)$ pdmog continued to circulate in the 2010/11 season as the predominant virus, but co-circulating with influenza $B$ viruses $[18,19]$. In Portugal, influenza B viruses were detected earlier in that season, in contrast to what was observed in Europe [18]. The circulation of influenza $A\left(\mathrm{H}_{1} \mathrm{~N}_{1}\right)$ pdmog virus occurred during the expected period without offseason peaks, but the peak of the 2010/11 season was registered by the Laboratory Network three weeks later than the peak observed by the sentinel surveillance system within the National Influenza Surveillance Programme (week 52, 2010, data not shown), which was in agreement with what was observed in Spain and the rest of Europe at that time [20].

The age group of 5-14 year-old children was most affected by $A\left(\mathrm{H}_{1} \mathrm{~N}_{1}\right)$ pdmog-associated influenza in $2009 / 10$, as was also observed in Europe [21,22]. However, in the 2010/11 season, the highest percentage of $A\left(\mathrm{H}_{1} \mathrm{~N}_{1}\right)$ pdmog-influenza positive cases was found in a broader age range, from 15 to 64 years. This was in agreement with what was observed in Austria and Norway, but in contrast to other European countries where the most affected age group were children under 15 years of age [19].

In the 2009/10 pandemic season, cough and contact with another influenza patients were strongly associated with confirmed $\mathrm{A}\left(\mathrm{H}_{1} \mathrm{~N}_{1}\right)$ pdmog influenza, whereas in $2010 / 11$, fever was the symptom that showed a higher association with a positive case. These results were consistent with other findings for seasonal [23] and pandemic influenza [24], where fever and cough were in strong association with influenza disease. This information can be useful for an earlier and more accurate diagnosis of influenza in order to maximise the effectiveness of antiviral therapy and to avoid unnecessary antibiotic use [23].

During the pandemic season, a genetically similarity of the strains in circulation with the A/California/7/2009 vaccine strain was observed. Since the $2010 / 11$ season, increased genetic variation has occurred among the pandemic $\mathrm{A}\left(\mathrm{H}_{1} \mathrm{~N}_{1}\right)$ pdmog viruses circulating in Portugal and in Europe [25]. The Portuguese viruses clustered into four different genetic clades from the eight genetic groups so far identified [26,27]. The majority of them clustered in genetic group 5 represented by the A/Astrakhan/1/2011 strain.

Despite this genetic variation among the circulating $A\left(\mathrm{H}_{1} \mathrm{~N}_{1}\right) p d m 09$, very little antigenic drift was observed over the two seasons, and isolates were closely related to the strain A/California/7/2009 included in the pandemic vaccine of the 2009/10 season and of the seasonal influenza vaccine for the $2010 / 11$ season.

During the two winter seasons, the Laboratory Network found two viruses with the $\mathrm{H}_{275} \mathrm{Y}$ amino acid substitution in neuraminidase that has been associated with 
oseltamivir resistance. Globally, a limited number of sporadic oseltamivir-resistant cases of $A\left(\mathrm{H}_{1} \mathrm{~N}_{1}\right)$ pdmog have been reported $[11,28]$, but the majority of circulating influenza $A\left(\mathrm{H}_{1} \mathrm{~N}_{1}\right)$ pdmog viruses were sensitive to neuraminidase inhibitors [11]. The two Portuguese $\mathrm{H} 275 \mathrm{Y}$ viruses were detected in immunocompromised patients under oseltamivir treatment: one was confirmed resistant to oseltamivir by phenotypic assays (2009/10 season); the other came from a case of severe influenza with fatal outcome (2010/11 season), but confirmation by phenotypic assays could not be done since the virus was not isolated. The monitoring of antiviral resistance was, and continues to be, essential since antiviral treatment is an important tool in the clinical management of severe or complicated influenza cases $[7,11]$.

During 2010/11 the Network also increased the diagnostic capability by implementing methodologies for the detection of other respiratory pathogens, having found several other agents associated with ILI co-circulating with influenza. This information can be a major contribution to a better understanding of the epidemiology of the disease.

Since September 2011 the Portuguese national health authorities have been developing and implementing an integrated surveillance system for SARI in five hospitals members of the Portuguese Laboratory Network. In the near future, this surveillance system will hopefully be extended to other hospitals and we expect that the established Laboratory Network will play a major role. Continuous laboratory participation and further advances in diagnostic capacity are essential to improve the surveillance of respiratory infections in Portugal.
Members of the Portuguese Laboratory Network for the Diagnosis of Influenza Infection:

Raquel Guiomar; Pedro Pechirra; Paulo Gonçalves; Ana Arraiolos; Patrícia Conde; Baltazar Nunes; Eleonora Paixão; Inês Batista; Cristina Furtado; Jorge Machado; Maria José Silvestre; Madalena Almeida Santos, Joana Sobrinho Simões; Maria do Rosário Costa; João Tiago Guimarães; Graça Ribeiro; João Vaz; Álvaro Beleza; Roger Oliveira; Aida Fernandes; Luís Milho; João Rego; Paula Luísa Fernandes; Graça Andrade; Luísa Mota Vieira; Rita Cabral Veloso; Jácome Bruges Armas; Ana Rita Pimentel Couto; Rute Marcelino; Teresa Porta Nova; José Melo Cristino; Dinah Carvalho; Carlos Ribeiro; Rosário Barreto; Virgínia Penim; Rita Corte Real; Paula Branquinho; Lurdes Gonçalves; Maria Helena Ramos; Ana Paula Castro; Filomena Caldeira; Manuel Maurílio.

\section{Acknowledgements}

We acknowledge the authors, originating and submitting laboratories of the sequences from GISAID's EpiFlu Database on which this research is based (see Table 1). All submitters of data may be contacted directly via the GISAID website www.gisaid.org

We acknowledge all the colleagues from the Department of Infectious Diseases (INSA) for their contribution to the laboratory diagnosis of influenza $\mathrm{A}\left(\mathrm{H}_{1} \mathrm{~N}_{1}\right)$ pdmog virus.

We acknowledge Dr. John McCauley and his staff from the WHO Collaborating Centre for Reference and Research on Influenza (National Institute for Medical Research) for the complementary antigenic analysis of our viral samples.

\section{References}

1. World Health Organisation (WHO). Manual for the laboratory diagnosis and virological surveillance of influenza. WHO Global Influenza Surveillance Network. Geneva: WHO; 2011. Available from: http://www.who.int/influenza/resources/documents/ manual diagnosis surveillance influenza/en/index.html

2. Zambon MC. Epidemiology and Pathogenesis of Influenza. J Antimicrob Chemother. 1999;44, Suppl B: 3-9.

3. Thompson WW, Shay DK, Weintraub E, Brammer L, Cox $\mathrm{N}$, Anderson LJ, et al. Mortality associated with influenza and Respiratory syncytial virus in the United States. JAMA. 2003;289(2): 179-86.

4. Nunes B, Viboud C, Machado A, Ringholz C, Rebelo-deAndrade $\mathrm{H}$, Nogueira P, et al. Excess Mortality Associated with Influenza Epidemics in Portugal, 1980 to 2004. PLoS One. 2011;6(6):e20661.

5. Amato-Gauci A, Zucs P, Snacken R, Ciancio B, Lopez V, Broberg $E$, et al. Surveillance trends of the 2009 influenza $A\left(\mathrm{H}_{1} \mathrm{~N}_{1}\right)$ pandemic in Europe. Euro Surveill. 2011;16(26):pii=19903. Available from: http://www.eurosurveillance.org/ViewArticle. aspx?Articleld $=19903$.

6. Rebelo de Andrade, H. Vigilância epidemiológica da gripe em Portugal. [Epidemiological surveillance of influenza in Portugal]. Rev Port Clin Geral. 2005;21:379-88. Portuguese.

7. Instituto Nacional de Saúde Dr. Ricardo Jorge (INSA). A gripe em Portugal nas épocas 2008/2009 e 2009/2010. Relatório do Programa Nacional de Vigilância da Gripe. [The influenza season in Portugal in 2008/2009 and 2009/2010. Report of the national influenza surveillance programme]. Lisbon: INSA; 2010. Portuguese. Available from: http://www.insa. $\mathrm{pt} /$ sites/INSA/Portugues/Publicacoes/Outros/Documents/ DoencasInfecciosas/Relatorio_PNVG_2008-2010.pdf

8. Direcção Geral da Saúde. Pandemia de Gripe. Plano de Contingência Nacional do Sector da Saúde para a Pandemia de Gripe. [National contingency plan for the health sector for pandemic influenza]. 2nd ed. Lisbon: 2008. Portuguese. Available from: http://www.dgs.pt/upload/membro.id/ ficheiros/i010835.pdf

9. Despacho nํㅜ $16548 / 2009$, de 21 Julho, Diário da República, $2^{a}$ série, № 139: 28507. Rede de laboratórios para o diagnóstico da infecção pelo vírus da gripe $\mathrm{A}\left(\mathrm{H}_{1} \mathrm{~N}_{1}\right) \mathrm{v}$. [Network of laboratories for the diagnosis of infection by the influenza virus $\left.A\left(\mathrm{H}_{1} \mathrm{~N} 1\right) \mathrm{v}\right]$. Portuguese. Available from: http://www. sg.min-saude.pt/NR/rdonlyres/134FD47F-1DF8-48DB-8912B64ACoF540D4/o/2850728507.pdf 
10. Instituto Nacional de Saúde Dr. Ricardo Jorge (INSA). Gripe Sazonal e Pandémica. [Seasonal and Pandemic influenza]. Lisbon: INSA; 2006. ISBN 978-972-8643-22-5. Portuguese.

11. World Health Organisation (WHO). Global Monitoring of antiviral resistance in currently circulating human influenza viruses. Wkly Epidemiol Rec. 2011; 86(45):497-508.

12. European Centre for Disease Prevention and Control (ECDC). Overview of Surveillance Influenza 2009/2010 in EU/EEA. Technical document. Stockholm: ECDC; 2009. Available from: http://www.ecdc.europa.eu/en/publications/ Publications/0909_TED_Overview_of_Surveillance_of_ Influenza_2009-2010_in_EU-EEA.pdf

13. Grist NR, Ross CA, Bell EJ. Diagnostic methods in clinical virology. 1st ed. Oxford: Blackwell Scientific Publishers; 1966.

14. World Health Organisation (WHO) Collaborating Centre for influenza at CDC Atlanta, United States. Sequencing primers and protocol. 12 May 2009 (first published 29 April 2009). Available from: http://www.who.int/csr/resources/ publications/swineflu/GenomePrimers_20090512.pdf

15. Tamura K, Peterson D, Peterson N, Stecher G, Nei M, Kumar S. MEGA5: molecular evolutionary genetics analysis using maximum likelihood, evolutionary distance, and maximum parsimony methods. Mol Biol Evol. 2011;28(10): 2731-9.

16. Nelson M, Spiro D, Wentworth D, Beck E, Fan J, Ghedin E, et al. The early diversification of influenza A/H1N1pdm. PLoS Curr. 2009; 1:RRN1126

17. Instituto de Salud Carlos III (ISCII).. Vigilância de la gripe en España. Evolución de la gripe pandémica por AnH1N1. (Desde la semana 20/2009 hasta la semana 20/2010). [Influenza surveillance in Spain. Evolution of pandemic influenza AnH1N1. (From week 20/2009 to week 20/2010)]. Spanish. Available from: http://www.isciii.es/ISCIII/es/contenidos/fd-servicioscientifico-tecnicos/fd-vigilancias-alertas/fd-enfermedades/ Vigilancia de la gripe en Espana Evolucion de la pandemia_por_AnH1N1_Temporada_2009-2010.pdf

18. World Health Organisation (WHO). Review of the 2010-2011 winter influenza season, northern Hemisphere. Wkly Epidemiol Rec. 2011; 86(22):222-7.

19. European Centre for Disease Prevention and Control (ECDC). Influenza Surveillance in Europe 2010-2011. Surveillance Report. Stockholm: ECDC; 2011. Available from: http:// ecdc.europa.eu/en/publications/Publications/111209_SUR_ Influenza_surveillance_Europe\%20_2010_2012.pdf.

20. Instituto de Salud Carlos III (ISCII). Vigilância de la gripe en España. Temporada 2010-11. (Desde la semana 40/2010 hasta la semana 20/2011). [Influenza surveillance in Spain. 2010-11 season. (From week 40/2010 to week 20/2011)]. Spanish. Available from: http://vgripe.isciii.es/gripe/ documentos/20102011/InformesAnuales/Informe_GRIPE_ Temporada_2010-11_07092011.pdf

21. Kelly H, Cowling B. Insights from Europe related to pandemic influenza $A\left(\mathrm{H}_{1} \mathrm{~N}_{1}\right) 2009$ have international relevance. Euro Surveill. 2011; 16(26):pii=19899. Available from: http://www. eurosurveillance.org/ViewArticle.aspx?Articleld=19899

22. Miller E, Hoschler K, Hardelid P, Stanford E, Andrews N, Zambon M. Incidence of 2009 pandemic influenza $\mathrm{A} \mathrm{H}_{1} \mathrm{~N}_{1}$ infection in England: a cross-sectional serological study. Lancet. 2010;375(9720): 1100-8.

23. Monto AS, Gravenstein S, Elliott M, Colopy M, Schweinle J. Clinical signs and symptoms predicting influenza infection. Arch Intern Med. 2000; 160(21): 3243-7.

24. Lochindarat S, Bunnag T. Clinical presentations of pandemic 2009 influenza $A\left(\mathrm{H}_{1} \mathrm{~N}_{1}\right)$ virus infection in hospitalized Thai children. J Medl Assoc of Thai. 2011;; 94 Suppl 3:S107-12.

25. World Health Organisation (WHO). Recommended composition of influenza virus vaccines for use in the 2011-2012 northern hemisphere influenza season. Wkly Epidemiol Rec. 2011; 86(10): 86-90

26. European Centre for Disease Prevention and Control (ECDC). Influenza virus characterisation. Summary Europe, August and September 2011. CNRL Technical document. Stockholm: ECDC 2011. Available from: http://ecdc.europa.eu/en/publications/ Publications/1110_SUR_Influenza_virus_characterization_ August_September\%202011.pdf

27. European Centre for Disease Prevention and Control (ECDC). Influenza virus characterisation. Summary Europe, March 2012. CNRL Technical document. . Stockholm: ECDC; 2012. Available from: http://www.ecdc.europa.eu/en/publications/ Publications/1204-TED-CNRL-report.pdf

28. European Centre for Disease Prevention and Control (ECDC). Oseltamivir-resistant influenza $A\left(\mathrm{H}_{1} \mathrm{~N}_{1}\right) 2009$ cluster in Australia. September 2011. CNRL Rapid Risk Assessment. Stockholm: ECDC; 2011. Available from: http://ecdc.europa. eu/en/publications/Publications/110906_TER_Rapid_Risk_ Assessment_Oseltamivir-resistant\%20influenza\%20A.pdf 